\title{
On the Performance of Hybrid RF/FSO Relay Algorithms for 5G Wireless Communication Systems
}

\author{
Vijay Kumar Tonk ${ }^{1}$ and Pradeep Kumar Yadav ${ }^{2}$ \\ ${ }^{1}$ Research Scholar \\ ${ }^{1}$ Uttarakhand Technical University, Dehradun, Uttrakhand (U.K.) India \\ ${ }^{2}$ Central Building Research Institute, Roorkee, Uttrakhand(U.K.), India \\ 1vijkrtonk@yahoo.com, ${ }^{2}$ prd_yadav@rediffmail.com
}

\begin{abstract}
Currently, free space optical (FSO) communication systems have become very popular because of its high achievable optical bandwidth, greater security against eavesdropping, low power consumption. In this paper, the authors have considered decode and forward (DF) Relay based dual hop hybrid radio frequency (RF) and FSO (RF/FSO) system. Where, RF link is modeled as Rayleigh faded distribution and FSO link is modeled as $\alpha-\mu$ distributed channel. A closed form expression of outage probability is derived for the proposed system. The effect of the channel parameters on the outage performance is also studied. Furthermore, we have also analyzed the asymptotic behavior of proposed analysis in high signal to noise ratio (SNR) regime. The analytical results are well aligned with asymptotic results, which shows the correctness of proposed analysis.
\end{abstract}

Keywords: RF/FSO, Relay, Decode and forward, $\alpha-\mu$ distribution, outage probability, Asymptotic analysis

\section{Introduction}

Wireless communication systems have gained lots of popularity in last two decades because of their ability to provide mobile access and unbounded connectivity. However, performance of wireless communication systems is degraded due to path loss, shadowing, and multipath fading effects. In multi-path fading phenomenon, two or more than two radio signals reaching at receiver by paths which arrives at slightly different times. These radio signals are called multi path signals, combined at the receiver. The resultant received signal can vary widely in terms of amplitude and phase [1]. The shadowing effect [2] is caused due to power absorption by obstacles present between the transmitting and receiving nodes. The path loss [1] is happened because of power dissipation effects of the propagation channel. Although, diversity combing using multiple antennas is one of the very popular techniques to mitigate deteriorative effect of multipath fading. Then also, due to the limitations of size and cost of wireless devices in sensor and cellular networks, placing multiple antennas on a single wireless node may not be practically possible. In such cases, cooperating with other nodes to form a distributed antenna system (DAS) becomes a more promising and desirable solution. This can be achieved using cooperative communications, where an intermediate node is place between transmitting and receiving node which is called relay. FSO Communication [2-11] uses optical carrier to convey information from one point to other through free space unguided channels. Due to its properties i.e. extremely high bandwidth, easy to deploy, unlicensed spectrum, low power consumption, reduced size and improved channel security, it is considered as an extension of frontier for high speed broadband connection. Secondary benefits of FSO systems are

Received (June 5, 2017), Review Result (September 3, 2017), Accepted (September 8, 2017) 
as: (i) easily expandable, (ii) light weight and compact in size, (iii) quick deploy ability, and (iv) can be deployed at places where optic fiber cables cannot be used. In [12-20] cooperative communication based free space optical (FSO) system has been studied. In [19], log-normal distribution is introduced to model the weak atmospheric turbulence in the FSO links. However, authors have been adopted the Gamma-Gamma distribution to represent wide range of atmospheric turbulence conditions (weak to strong). Authors in [12-17] have proposed a cooperative diversity technique to mitigate the effect of fading over the radio frequency (RF) channel. In [13, 17-20], outage performance is analyzed over weak, moderate and strong turbulence. Relay-aided FSO system is analyzed by Wang et. al in [21]. Performance metrics is very important to monitor any system's operation, and so metrics such as BER outage probability become very useful. Point-topoint transmission has been analyzed in the past but according to authors, when the distance between transmitters and receivers is increased in kilometers, then relay operation must be utilized. Wang et. al., have analyzed relay assisted network for serial and parallel transmission. Outage probability has been calculated and plotted against transmitted power. In order to compare relay FSO with direct transmission system, authors have also evaluated outage probability for directly connected (or P2P) FSO systems. Polarization has been opted as modulation technique in FSO systems [22]. Tang et. al have reported coherent binary polarization shift keying (BOLSK) for reduction of BER in turbulence effected FSO systems. When larger receiver apertures are replaced by several smaller apertures, received wave front becomes coherent, thus increasing reception quality. Based on this fact, authors have proposed BOLSK-MIMO FSO system. In the aforementioned literature, the outage probability and bit error rate (BER) performance is analyzed for one-way relaying (OWR). The spectral efficiency of the system can further be improved by using two-way relay (TWR) in place of OWR. In [23], authors have proposed two relay selection for multi relay FSO network. Yacoub in [24], proposed $\alpha-\mu$ distributed random variable includes several distributions as special cases such as Nakagami-m, Rayleigh and Weibull distribution. Peppas [25] demonstrates the utilization of $\alpha-\mu$ distribution as a general fading model for FSO systems. In this paper, we have analyzed the outage performance analysis for decode and forward relay scheme over asymmetric RF/FSO fading channel. The rest of paper is arranged as follows: Section II describes proposed system model. In Section III, a closed form expressions of outage probability (OP) is derived. Numerical results are discussed in Section IV and finally the paper is concluded and summarized in Section V.

\section{System Model}

In this research work, we consider dual hop decode and forward relay network as illustrated in Figure 1. For given scenario, in first hop, data is transmitted between source node $\mathrm{S}$ and relay node $\mathrm{R}$. While in second hop, relay node $\mathrm{R}$ decodes the received signal from $S$ and forwards to destination D. Considering there is no direct link between $S$ and D. The fading characteristics of the S-R link is modeled as Rayleigh distribution where the received signal at the relay node is expressed as

$$
r=\alpha_{1} x+n_{1}
$$

where $\alpha_{1}$ is Rayleigh fading coefficient, $x$ is the signal input at relay node and $n_{1}$ is additive white Gaussian noise (AWGN).

The probability density function (PDF) of Rayleigh fading channel in terms of instantaneous SNR can be expressed as

$$
f_{S R}(\gamma)=\frac{1}{\overline{\gamma_{S R}}} \exp \left(-\frac{\gamma}{\overline{\gamma_{S R}}}\right)
$$


Where $\overline{\gamma_{S R}}$ is the average SNR between S-R link.

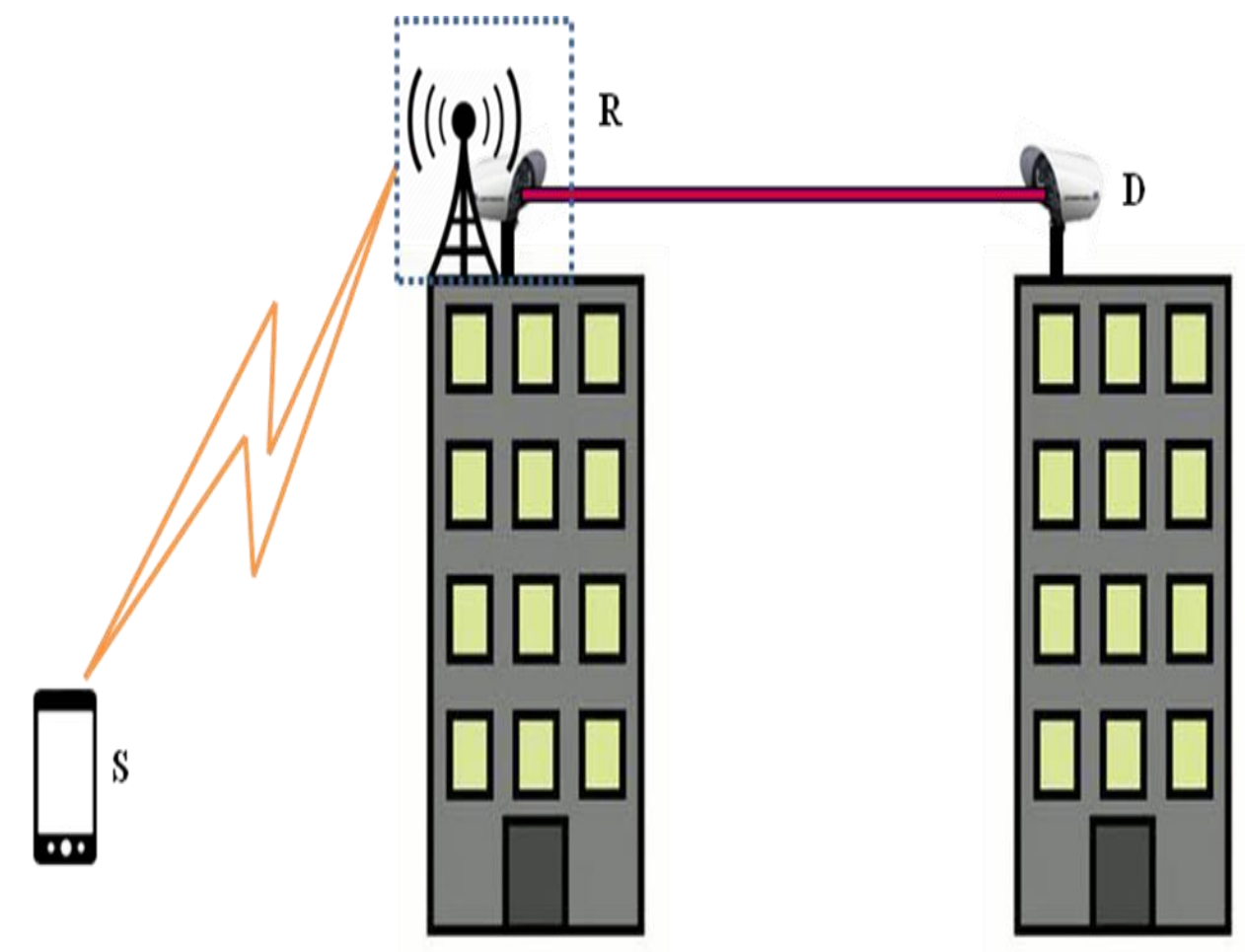

Figure 1. System Model

On the other hand, the probability density function (pdf) of the R-D link has been modelled as $\alpha-\mu$ distributed random variable, as given by [24]

$$
f_{R D}(\gamma)=\frac{\alpha \mu^{\mu} \gamma^{\left(\frac{\alpha \mu}{2}-1\right)}}{2 \Gamma(\mu) \overline{\gamma_{R D}}} \exp \left(-\mu\left(\frac{\gamma}{\overline{\gamma_{R D}}}\right)^{\frac{\alpha}{2}}\right)
$$

where $\alpha$ is an arbitrary fading parameter, $\mu$ is the inverse of the normalized variance defined as [13]

$$
\mu=\frac{E^{2}\left[R_{\alpha}\right]}{V\left[R_{\alpha}\right]}
$$

where $\mathrm{E}$ and $\mathrm{V}$ are the expectation and the variance operators, respectively.

\section{Outage Probability Analysis}

Outage probability (OP) is an important performance evaluation metric, OP, measures the probability of failing to achieve an output SNR threshold required for a desired service. In this section, the performance of proposed system is evaluated in terms of outage probability (OP). The outage probability can be expressed [8], as: 


$$
\begin{aligned}
P_{\text {out }} & =P_{r}\left\{\min \left(\gamma_{S R}, \gamma_{S D}\right)<\gamma_{t h}\right\} \\
& =F_{S R}\left(\gamma_{t h}\right)+F_{R D}\left(\gamma_{t h}\right)-F_{S R}\left(\gamma_{t h}\right) F_{R D}\left(\gamma_{t h}\right)
\end{aligned}
$$

where $F(\circ)$ represents Cumulative density function.

$$
F_{S R}\left(\gamma_{t h}\right)=\int_{0}^{\gamma_{t h}} f_{S R}(\gamma) d \gamma
$$

By substituting of sin (5), The above integration can be expressed as

$$
F_{S R}\left(\gamma_{t h}\right)=\frac{1}{\overline{\gamma_{S R}}} \int_{0}^{\gamma_{t h}} \exp \left(-\frac{\gamma}{\overline{\gamma_{S R}}}\right) d \gamma
$$

After some mathematical manipulation, the above integral can be written as

$$
F_{S R}\left(\gamma_{t h}\right)=1-\exp \left(-\frac{\gamma_{t h}}{\overline{\gamma_{S R}}}\right)
$$

Similarly, as above, by su

$$
F_{R D}\left(\gamma_{t h}\right)=\frac{\alpha \mu^{\mu}}{2 \Gamma(\mu) \overline{\gamma_{R D}}} \int_{0}^{\gamma_{t h}} \gamma^{\left(\frac{\alpha \mu}{2}-1\right)} \exp \left(-\mu\left(\frac{\gamma}{\overline{\gamma_{R D}}}\right)^{\frac{\alpha}{2}}\right) d \gamma
$$

The above expression can be solved using [26, (3.381.8)], The resultant expression can be written as:

$$
F_{R D}\left(\gamma_{t h}\right)=\frac{1}{\Gamma(\mu)} \gamma\left(\mu,\left(\frac{\gamma_{t h}}{\overline{\gamma_{R D}}}\right)^{\frac{\alpha}{2}}\right)
$$

where $\gamma(\circ)$ represents the lower incomplete gamma function as given in [15 ].

By substituting value from equation (7) and (8) in equation (4), The resultant value of outage probability $\left(P_{\text {out }}\right)$ can be written as:

$$
P_{\text {out }}=1-\exp \left(-\frac{\gamma_{\text {th }}}{\overline{\gamma_{S R}}}\right)+\frac{1}{\Gamma(\mu)} \gamma\left(\mu,\left(\frac{\gamma_{\text {th }}}{\overline{\gamma_{R D}}}\right)^{\frac{\alpha}{2}}\right)-\frac{1}{\Gamma(\mu)}\left(1-\exp \left(-\frac{\gamma_{t h}}{\overline{\gamma_{S R}}}\right)\right)\left(\mu,\left(\frac{\gamma_{\text {th }}}{\overline{\gamma_{R D}}}\right)^{\frac{\alpha}{2}}\right)
$$

\section{Asymptotic Analysis}

In order to get better insight of attained expression of outage probability. Asymptotic expression of in outage probability in high SNR $(\bar{\gamma} \rightarrow \infty)$ has been derived. The expression of outage probability in high SNR, equation (4) can be expressed as [27] 


$$
P_{\text {out }}\left(\gamma_{t h}\right) \approx F_{S R}\left(\gamma_{t h}\right)+F_{R D}\left(\gamma_{t h}\right)
$$

By putting $\bar{\gamma}_{R D}=\bar{\gamma}_{S D}=\bar{\gamma}$ and setting $\bar{\gamma} \rightarrow \infty$,

From equation (7), $F_{S R}\left(\gamma_{t h}\right)=1-\exp \left(-\frac{\gamma_{t h}}{\bar{\gamma}}\right) \rightarrow 0$ for $\bar{\gamma} \rightarrow \infty$

Similarly from equation (8), $F_{R D}\left(\gamma_{t h}\right)=\frac{1}{\Gamma(\mu)} \gamma\left(\mu,\left(\frac{\gamma_{t h}}{\bar{\gamma}}\right)^{\frac{\alpha}{2}}\right)$ for $\bar{\gamma} \rightarrow \infty$ and using the equation from $[26,(8.354 .1)]$ and neglecting higher order terms, the above equation can be expressed as :

$$
F_{R D}\left(\gamma_{t h}\right)=\frac{1}{\Gamma(\mu)}\left(\frac{\gamma_{t h}}{\bar{\gamma}}\right)^{\frac{\alpha \mu}{2}}
$$

By substituting results, from equation (11) and equation (12) in equation (10), the resultant expression of outage probability can be expressed as :

$$
P_{\text {out }}\left(\gamma_{t h}\right)=\frac{1}{\Gamma(\mu)}\left(\frac{\gamma_{t h}}{\bar{\gamma}}\right)^{\frac{\alpha \mu}{2}}
$$

\section{Results and Discussion}

In this section, the analytical results for the outage probability for the DF based dual hop hybrid RF/FSO system, without considering the pointing error has been discussed. Here RF link is modeled as Rayleigh distribution and the FSO link is modeled as $\alpha-\mu$ distribution.

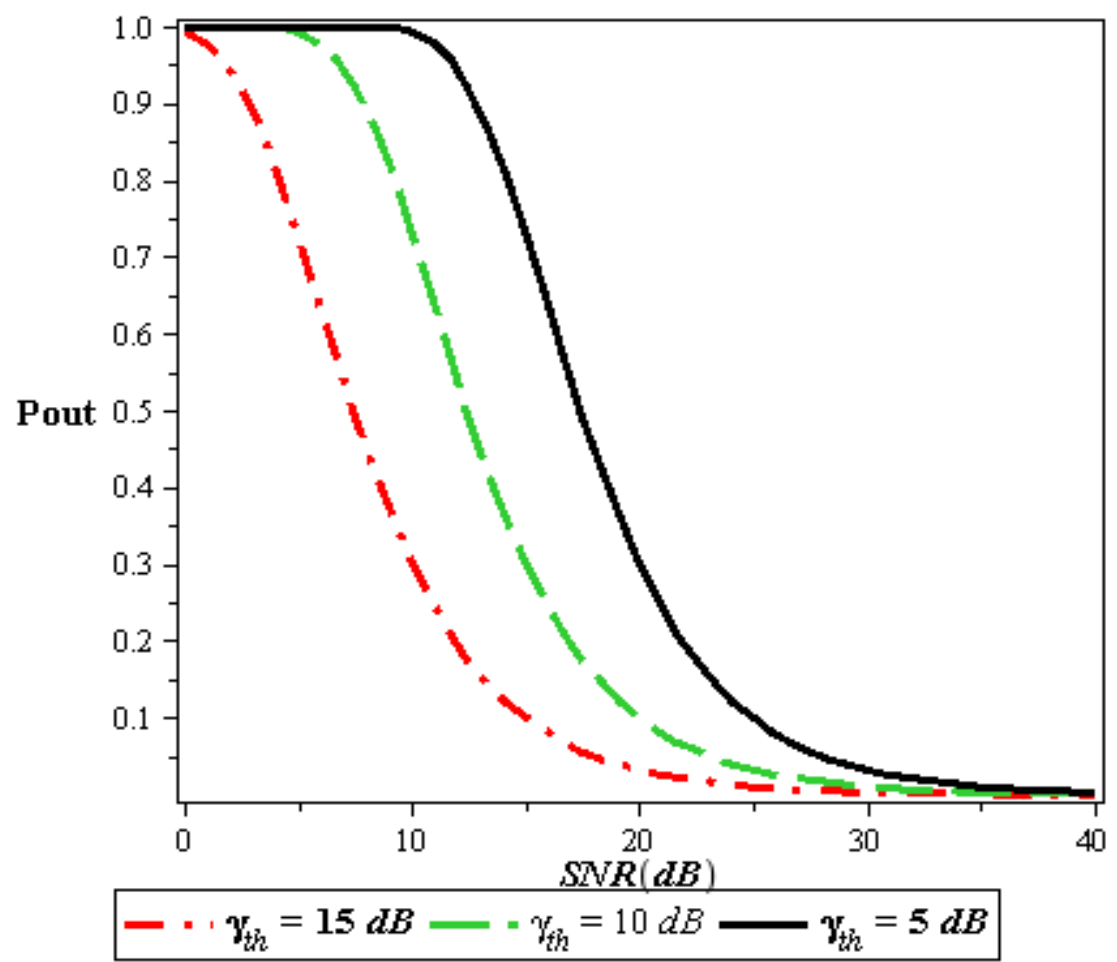

Figure 2. Outage Probability versus SNR for Various Values Threshold 


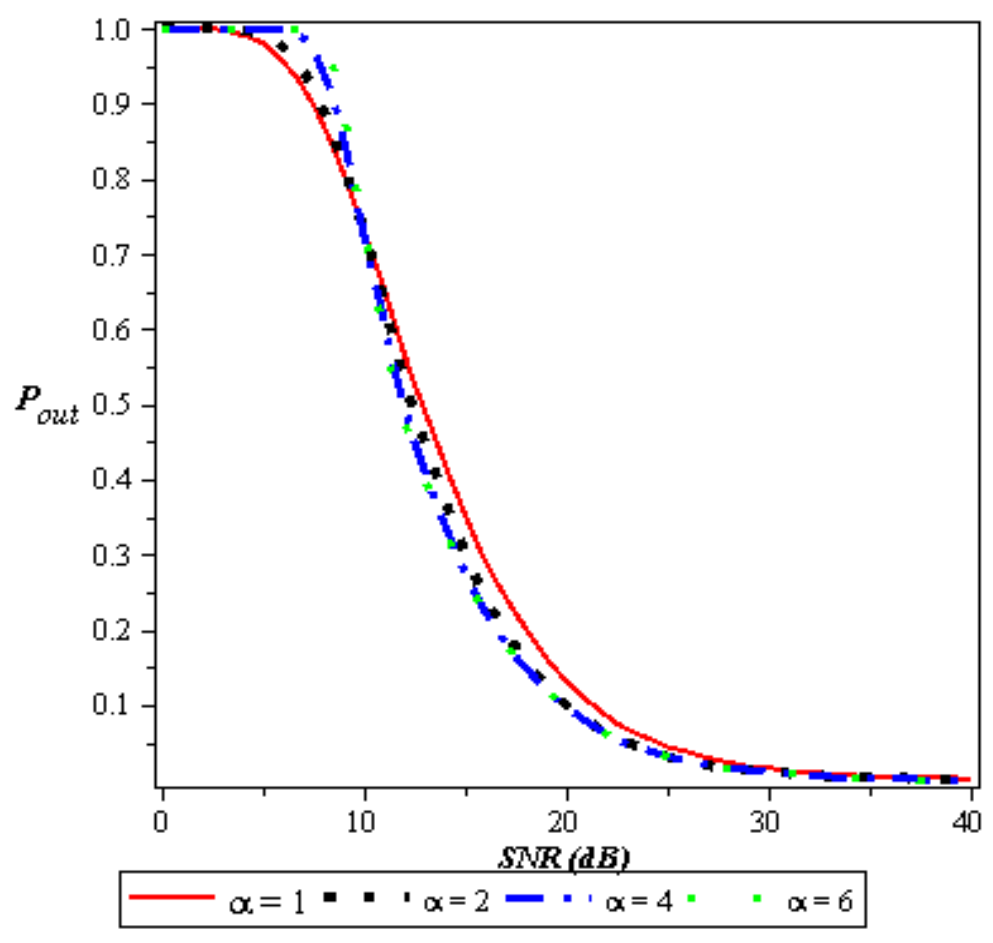

Figure 3. Outage Probability versus SNR for Various Values Channel Parameter $(\alpha)$

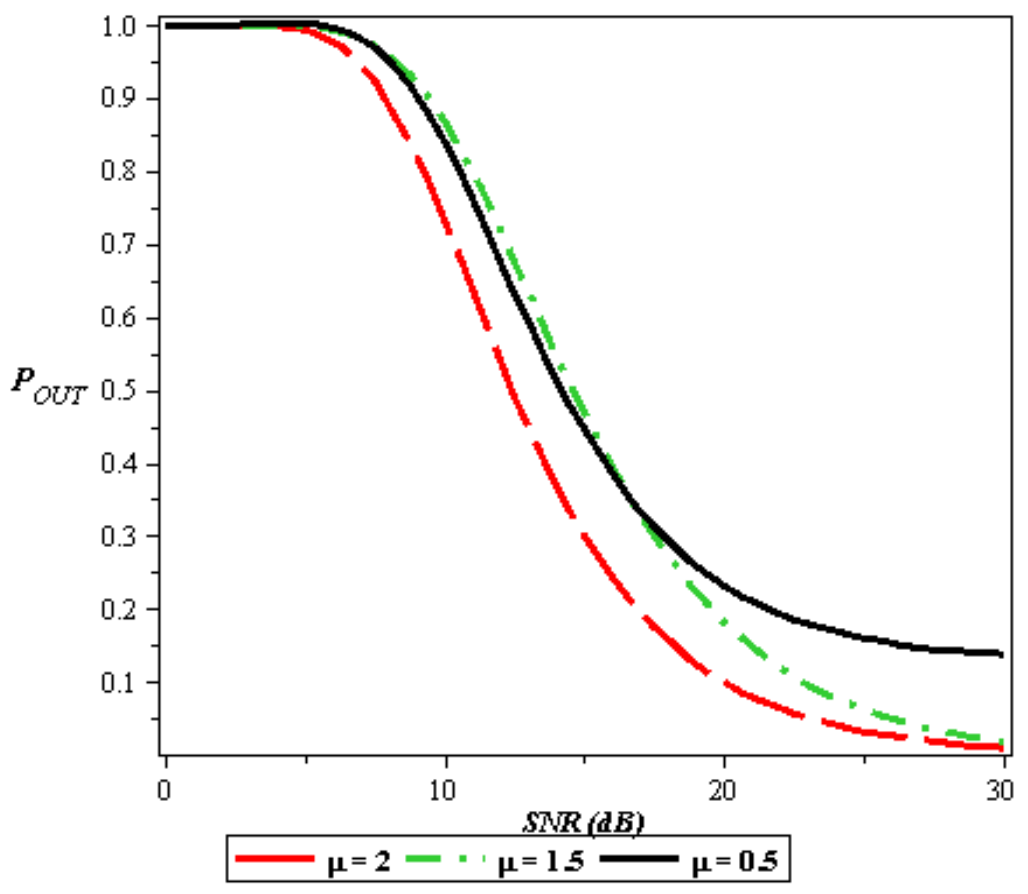

Figure 4. Outage Probability versus SNR for Various Values Channel Parameter $(\mu)$

Figure 2, shows Outage probability versus SNR plot for various values threshold SNR. For plot of Figure 2, we consider $\alpha=2$ and $\mu=2$. It is clear from the Figure that Outage probability improves with increase of SNR. Outage probability also improves with the 
increase of threshold SNR. Figure 3 shows Outage probability versus SNR for various values channel parameter. It can be observed from the Figure,

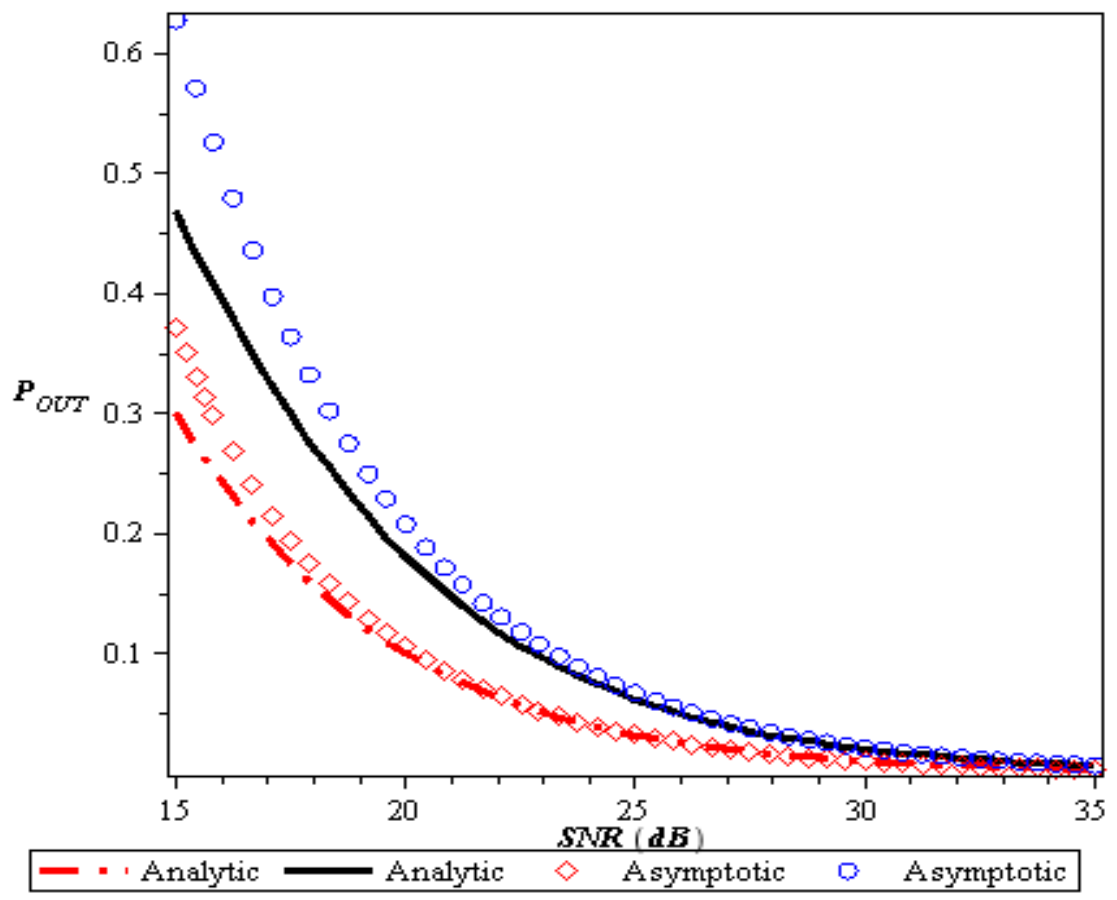

Figure 5. Outage Probability versus SNR

that Outage probability improves with increase of SNR but it does not changes much with the channel of parameter $\alpha$. Figure 4 shows Outage probability versus SNR for various values channel parameter $(\mu)$. It is clear from the Figure that improves with increase of SNR. Moreover, Outage probability also improves with the increase channel parameter $(\mu)$. Figure 5 shows asymptotic behavior outage probability of proposed analysis. It is clear from Figure that asymptotic results are well match with analytical results in high SNR regime which shows the correctness of proposed analysis.

\section{Conclusions}

In this research work, we present the mathematical modeling of a dual hop hybrid RF/FSO DF relaying system. More specifically, we provide closed loop expressions for outage probability for the proposed system. Moreover, we also show the effect of variation of various fading and channel parameters on the outage probability. In addition, we have also analyzed the asymptotic behavior proposed analysis which verifies the accuracy of proposed analysis. This technique may be useful for future wireless communication such as $5 \mathrm{G}$ wireless communication system.

\section{References}

[1] T. S. Rappaport, "Wireless communications: principles and practice", New Jersey: prentice hall PTR, (1996).

[2] M. A. Khalighi and M. Uysal, "Survey on Free Space Optical Communication: A Communication Theory Perspective", IEEE Communications Surveys \& Tutorials, vol. 16, no. 4, (2014), pp. 2231-2258.

[3] E. Zedini, I. S. Ansari and M. S. Alouini, "Performance Analysis of Mixed Nakagami-m and Gamma Gamma Dual-Hop FSO Transmission Systems", IEEE Photonics Journal, vol. 7, no. 1, (2015), pp. 1-20.

[4] H. G. Sandalidis, T. A. Tsiftsis, G. K. Karagiannidis and M. Uysal, "BER Performance of FSO Links over Strong Atmospheric Turbulence Channels with Pointing Errors", IEEE Communications Letters, vol. 12, no. 1, (2008), pp. 44-46. 
[5] Y. Tang, M. Brandt-Pearce and S. G. Wilson, "Adaptive coding and modulation for hybrid FSO/RF systems", 2009 Conference Record of the Forty-Third Asilomar Conference on Signals, Systems and Computers, Pacific Grove, CA, (2009), pp. 1644-1649.

[6] L. Yang, X. Gao and M. S. Alouini, "Performance Analysis of FreeSpace Optical Communication Systems With Multiuser Diversity Over Atmospheric Turbulence Channels", in IEEE Photonics Journal, vol. 6, no. 2, (2014), pp. 1-17.

[7] I. S. Ansari, F. Yilmaz and M. S. Alouini, "Impact of Pointing Errors on the Performance of Mixed RF/FSO Dual-Hop Transmission Systems", IEEE Wireless Communications Letters, vol. 2, no. 3, (2013), pp. 351-354.

[8] I. S. Ansari, F. Yilmaz and M. S. Alouini, "Performance Analysis of Free-Space Optical Links Over M" alaga (M) Turbulence Channels With Pointing Errors", IEEE Transactions on Wireless Communications, vol. 15 , no. 1, (2016), pp. 91-102.

[9] T. A. Tsiftsis, H. G. Sandalidis, G. K. Karagiannidis and N. C. Sagias, "Multihop Free-Space Optical Communications Over Strong Turbulence Channels", IEEE International Conference on Communications, Istanbul, (2006), pp. 2755-2759.

[10] X. Tang, Z. Wang, Z. Xu and Z. Ghassemlooy, "Multihop Free-Space Optical Communications Over Turbulence Channels with Pointing Errors using Heterodyne Detection", Journal of Light wave Technology, vol. 32, no. 15, (2014), pp. 2597-2604.

[11] E. Zedini and M. S. Alouini, "On the Performance of Multihop Heterodyne FSO Systems With Pointing Errors", IEEE Photonics Journal, vol. 7, no. 2, (2015), pp. 1-10

[12] M. R. Bhatnagar, "Performance Analysis of Decode-and-Forward Relaying in Gamma-Gamma Fading Channels", IEEE Photonics Technology Letters, vol. 24, no. 7, (2012), pp. 545-547.

[13] L. Yang, X. Gao and M. Alouini, "Performance Analysis of Relay Assisted All-Optical FSO networks Over Strong Atmospheric Turbulence Channels with Pointing Errors", Journal of Lightwave Technology, vol. 32, no. 23, (2014), pp. 4011-4018.

[14] N. D. Chatzidiamantis, D. S. Michalopoulos, E. E. Kriezis, G. K. Karagiannidis and R. Schober, "Relay Selection Protocols for Relay-Assisted Free-Space Optical Systems", IEEE/OSA J. Opt. Commun. Netw., vol. 5, no. 1, (2013), pp. 92-103.

[15] R. B. Ruiz, A. G. Zambrana, B. C. Vazquez and C. C. Vazquez, "Impact of relay placement on diversity order in adaptive selective DF relay-assisted FSO communications", Opt. Exp., vol. 23, no. 3, (2015), pp. $2600-2617$.

[16] M. Safari and M. Uysal, "Relay-Assisted Free-Space Optical Communication”, IEEE Trans. Wireless Communication, vol. 7, no. 12, (2008), pp. 5441-5449.

[17] S. Anees and M. R. Bhatnagar, "Performance of an Amplify-and-Forward Dual-Hop Asymmetric RFFSO Communication System", IEEE/OSAJ. Opt. Communication Network, vol. 7, no. 2, (2015), pp. 124-135.

[18] M. Niu, J. Cheng and J. F. Holzman, "Error Rate Performance Comparison of Coherent and Subcarrier Intensity Modulated Optical Wireless Communications", IEEE/OSA J. Opt. Communication and Network, vol. 5, no. 6, (2013), pp. 554-564.

[19] A. A. Farid and S. Hranilovic, "Outage Capacity Optimization for Free-Space Optical Links with Pointing Errors", IEEE/OSA J. Lightwave Technology, vol. 25, no. 7, (2007), pp. 1702-1710.

[20] B. Rankov and A. Wittneben, "Spectral Efficient Protocols for Half Duplex Fading Relay Channels", IEEE Journal on Selected Areas Communication, vol. 25, no. 2, (2007), pp. 379-389.

[21] J. Y. Wang, J. B. Wang, M. Chen, Y. Tang and Y. Zhang, "Outage Analysis for Relay-Aided FreeSpace Optical Communications Over Turbulence Channels With Nonzero Boresight Pointing Errors", in IEEE Photonics Journal, vol. 6, no. 4, (2014), pp. 1-15.

[22] X. Tang, Z. Xu and Z. Ghassemlooy, "Coherent Polarization Modulated Transmission through MIMO Atmospheric Optical Turbulence Channel", in Journal of Lightwave Technology, vol. 31, no. 20, (2013), pp. 3221-3228.

[23] P. Puri, N.D. Chatzidiamantis, P. Garg, M. Aggarwal and G. K. Karagiannidis, "Two-Way Relay Selection in Multiple Relayed FSO Networks", IEEE Wireless Communication Letter, vol. 4, no. 5, (2015).

[24] M. D. Yacoub, “The $\alpha-\mu$ Distribution: A Physical Fading Model for the Stacy Distribution”, IEEE Transactions on Vehicular Technology, vol. 56, no. 1, (2007), pp. 27-34.

[25] K. P. Peppas, "A Simple, Accurate Approximation to the Sum of Gamma Gamma Variates and Applications in MIMO Free-Space Optical Systems", IEEE Photonics Technology Letters, vol. 23, no. 13, (2011).

[26] I. S. Gradshteyn and I. M. Ryzhik, "Table of Integrals, Series and Products", New York, NY, USA: Academic, (2000).

[27] L. Yang, X. Gao and M. S. Alouini, "Performance Analysis of Relay-Assisted All-Optical FSO Networks Over Strong Atmospheric Turbulence Channels With Pointing Errors", in Journal of Lightwave Technology, vol. 32, no. 23, (2014), pp. 4613-4620. 


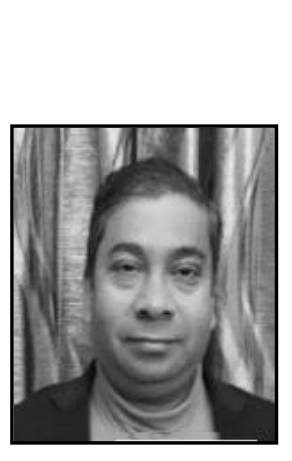

\section{Authors}

Vijay Kumar Tonk, he was born on 14 October, 1971 at Dehradun. His earlier education has been from DehraDun. After Schooling he completed his B.Sc. in 1992 from SGRR (PG) College, (HNB Garhwal University) in Maths, Physics \& Chemistry subjects. In 1996, he completed his Master of Computer Applications (MCA) from Gurukul Kangri Vishwavidyalaya, Haridwar. Presently, he is working as Lecturer (Computer Applications), Meera Bai Institute of Technology, (under DTTE Govt. of NCT of Delhi) New Delhi -110065, puruing his Ph.D. from Uttrakhand Technical University, Dehradun. Life member of Computer Society of India. And Student member of IEEE Computer Society.The areas of his interest are Wireless communication, Distributed Computing Systems, Task Allocation in Distributed Environment and Computer Network Security.

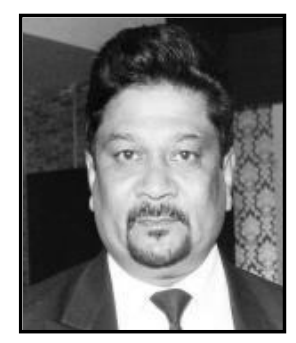

P. K. Yadav, he was born in Roorkee in 1963. He did his M.Sc.(1983) and Ph. D. (1996) from Gurukul Kangri Vishwavidyalaya, Haridwar. Application of Mathematical Modeling in Building Science \& Technology, Distributed Computing System, Grid Computing, Network Security, and Fire Modeling are the areas of his research. He started his career as Junior Scientific Assistant in 1984 and presently working as Principle Scientific Officer in CSIR-Central Building Research Institute. Dr. Yadav also holds the position as Associate Professor in the Discipline of Mathematical \& Information Sciences in Academy of Scientific \& Innovative Research (AcSIR). Dr Yadav has been awarded Shri M. S. Jain Beinel Memorial Award for Innovation Piling and other Construction Techniques by Indian Geo Technical Society and Golden Jubilee Director Award from CSIR-CBRI Roorkee. He has the life membership of many professional Societies and reviewed several research papers for the different reputed journals in the area of computer science and soft computing technique. He has contributed in many R \& D projects in the field of Building Science Technology and more than one hundred research publications are to his credit. He has supervised 8 $\mathrm{Ph}$. D students and four are pursuing Ph. D under him. 
International Journal of Future Generation Communication and Networking Vol. 10, No. 10 (2017) 